Article

\title{
A Trace Carbon Monoxide Sensor Based on Differential Absorption Spectroscopy Using Mid-Infrared Quantum Cascade Laser
}

\author{
Chen Chen ${ }^{\circ}$, Qiang Ren, Heng Piao, Peng Wang and Yanzhang Wang * \\ College of Instrumentation \& Electrical Engineering, Key Laboratory of Geophysical Exploration Equipment, \\ Ministry of Education of China, Jilin University, Changchun 130026, China; cchen@jlu.edu.cn (C.C.); \\ renqiang15@mails.jlu.edu.cn (Q.R.); piaoheng18@mails.jlu.edu.cn (H.P.); wangpeng18@mails.jlu.edu.cn (P.W.) \\ * Correspondence: yanzhang@jlu.edu.cn
}

Received: 25 November 2018; Accepted: 14 December 2018; Published: 18 December 2018

\begin{abstract}
Carbon monoxide (CO), as a dangerous emission gas, is easy to accumulate in the complex underground environment and poses a serious threat to the safety of miners. In this paper, a sensor using a quantum cascade laser with an excitation wavelength of $4.65 \mu \mathrm{m}$ as the light source, and a compact multiple reflection cell with a light path length of $12 \mathrm{~m}$ is introduced to detect trace $\mathrm{CO}$ gas. The sensor adopts the long optical path differential absorption spectroscopy technique (LOP-DAST) and obtains minimum detection limit (MDL) of $108 \mathrm{ppbv}$ by comparing the residual difference between the measured spectrum and the Voigt theoretical spectrum. As a comparison, the MDL of the proposed sensor was also estimated by Allan deviation; the minimum value of $61 \mathrm{ppbv}$ is achieved while integration time is $40 \mathrm{~s}$. The stability of the sensor can reach $2.1 \times 10^{-3}$ during the $2 \mathrm{~h}$ experimental test and stability of $1.7 \times 10^{-2}$ can still be achieved in a longer $12 \mathrm{~h}$ experimental test.
\end{abstract}

Keywords: Trace carbon monoxide sensor; mid-infrared spectrum; quantum cascade laser; differential absorption spectroscopy; residual analysis

\section{Introduction}

Compared to traditional bipolar semiconductor lasers, quantum cascade lasers (QCLs) offer unique advantages of good monochromaticity, high quantum efficiency, good temperature stability, flexible wavelength design, and fast response [1-3]. In addition, the infrared spectrum of QCL covers three important atmospheric transmission windows. Therefore, QCLs have an incomparable advantage over other luminous sources in the field of gas detection [4-6].

In recent years, the application of QCL to detect trace gas in the infrared "fingerprint region" has been developing rapidly. In 2008, J. B. Mcmanus et al. at the Aerodyne research center, adopted a QCL with center wave number of $967 \mathrm{~cm}^{-1}$ combined with a long optical path direct absorption method to detect ammonia gas. The light path length of multiple reflecting cell was increased to $76 \mathrm{~m}$, and the minimum detection limit (MDL) of ammonia gas was achieved as $0.2 \mathrm{ppbv}$ [7]. In 2013, P. G. Carbajo et al. detected $\mathrm{H}_{2} \mathrm{CO}$ using a QCL with central wave number of $1769 \mathrm{~cm}^{-1}$. The MDL was improved to $0.06 \mathrm{ppbv}$ by using the optical feedback-cavity enhanced absorption spectrum technology [8]. In 2010, K. Ruifeng et al. utilized the pulsed QCL with the central wave number of $1904 \mathrm{~cm}^{-1}$ combined with direct absorption spectrum detection technology to detect nitric oxide gas [9]. The MDL of nitric oxide reached $3.4 \mathrm{ppmv}$ by performing the least squares calculation method to fit baseline. In 2014, L. Guolin et al. reported a $\mathrm{CO}_{2}$ sensor using the $4.8 \mu \mathrm{m}$ mid-infrared QCL to realize a MDL of $180 \mathrm{ppbv}$ [10].

The optimization work on open gas cell using ellipsoid condensers is the main contribution here. Although the mentioned sensors achieved good detection performance for target gases, the shortcoming 
of complicated structure limits their usage in field applications. In this paper, a new CO sensor using mid-infrared QCL with center wavelength of $4.65 \mu \mathrm{m}$ is introduced. The sensor utilizes a $12 \mathrm{~m}$ long optical path differential absorption spectroscopy technique (LOP-DAST) to achieve MDL of $108 \mathrm{ppbv}$, minimum Allan deviation of $61 \mathrm{ppbv}$, and high performance of working stability during the long experimental test. To resolve the issue of large packages of core constituted components, chip-level mid-infrared gas sensors with high sensitivity is discussed in the final section, which appears very promising for future gas sensing.

\section{Detection Principle of CO Using LOP-DAST}

\subsection{Selection of CO Absorption Line}

In the mid-infrared spectrum band, the absorption spectrum of gas molecules generally includes rotational spectrum and rotational vibration spectrum, and each gas has many absorption bands in the mid-infrared spectrum band [11-13]. Taking $\mathrm{CO}$ as a target, the absorption spectrum band around $4.6 \mu \mathrm{m}$ caused by the fundamental frequency vibration of $\mathrm{CO}$ molecules is shown in Figure 1.

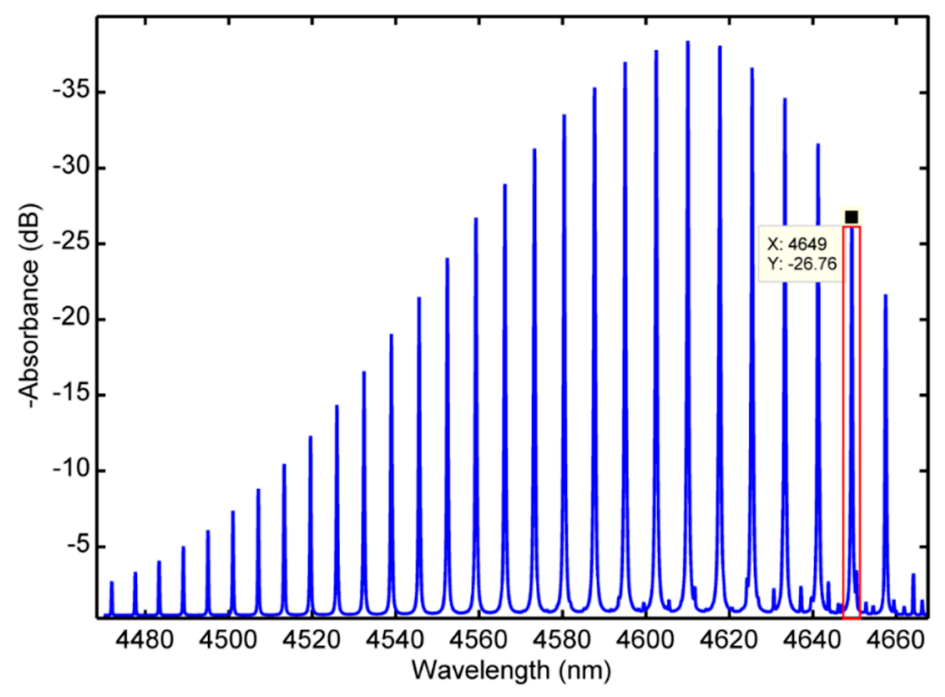

Figure 1. Absorption spectrum of CO.

As shown in Figure 1, the $\mathrm{x}$ coordinate is wavelength (nanometers, $\mathrm{nm}$ ), the y coordinate is absorption intensity (decibel, $\mathrm{dB}$ ), and blue lines represent $\mathrm{CO}$ gas absorption lines. Due to the limitation of tuning ability of mid-infrared QCL adopted in this paper, the optimum luminous wavelength is chosen at $4.65 \mu \mathrm{m}$, which is close to the peak value of $\mathrm{CO}$ absorption spectrum at $4.649 \mu \mathrm{m}$, as illustrated in the red box. For this absorption spectrum line, the absorption coefficient of $\mathrm{CO}$ is $-26 \mathrm{~dB}$ and is in the atmospheric window range. In addition, the improved accuracy of measured results can be reached because of spectral interference exclusion from other atmospheric components, such as water, methane, and $\mathrm{CO}_{2}$.

\subsection{Derivation of LOP-DAST technique}

The LOP-DAST can be described as follows. According to the Beer-Lambert law, absorbance is proportional to the concentrations of the attenuating species in the material sample [14-18]. The light intensity after passing through the gas absorption cell with light path length $L$ is:

$$
I(\lambda)=I_{0}(\lambda) \exp \left(\phi\left(\sigma_{i}, C_{i}, L, \varepsilon_{r}, \varepsilon_{m}\right)\right)
$$


The multiple variables function in the above equation can be expressed as:

$$
\phi\left(\sigma_{i}, C_{i}, L, \varepsilon_{r}, \varepsilon_{m}\right)=\sum_{i=1}^{n} \sigma_{i}(\lambda) C_{i} L+\varepsilon_{r}(\lambda)+\varepsilon_{m}(\lambda)
$$

Here, $\lambda$ is the wavelength of luminous light, $\sigma_{i}(\lambda)$ denotes the absorption cross section of the $i$ th gas, $C_{i}$ is the average concentration of the $i$ th gas, $L$ is the light path length, $\varepsilon_{r}(\lambda)$ is Rayleigh scattering, $\varepsilon_{m}(\lambda)$ is Mie scattering. The absorption cross section of Rayleigh scattering and Mie scattering are shown as a broadband absorption cross section. The LOP-DAST method decomposes the absorption cross section into broadband absorption cross section and narrow absorption cross section, namely:

$$
\sigma_{i}(\lambda)=\sigma_{i}^{b}(\lambda)+\sigma_{i}^{\prime}(\lambda)
$$

where, $\sigma_{i}^{b}(\lambda)$ represents the broadband absorption cross section of the $i$ th gas, and $\sigma_{i}^{\prime}(\lambda)$ represents the narrow absorption cross section of the $i$ th gas. Therefore, Formula (2) can be divided into the following form:

$$
\begin{gathered}
\phi^{b}\left(\sigma_{i}^{b}, C_{i}, L, \varepsilon_{r}, \varepsilon_{m}\right)=\sum_{i=1}^{n} \sigma_{i}^{b}(\lambda) C_{i} L+\varepsilon_{r}(\lambda)+\varepsilon_{m}(\lambda) \\
\phi^{\prime}\left(\sigma_{i}^{\prime}, C_{i}, L\right)=\sum_{i=1}^{n} \sigma_{i}^{\prime}(\lambda) C_{i} L
\end{gathered}
$$

By means of numerical filtering, the terms $\phi^{b}$ only containing broadband absorption cross section can be removed, so as to obtain the differential optical density of the measured gas,

$$
\text { (O.D. })_{\lambda}=\ln \left[\frac{I(\lambda)}{I_{0}(\lambda)}\right]=\sum \sigma_{i}^{\prime}(\lambda) C_{i} L
$$

The CO concentration in absorption cell can be obtained by fitting the differential optical density with the reference spectrum via the least square method.

The above analysis shows that obtaining the reference absorption cross section in the absorption spectrum band is key to measuring the $\mathrm{CO}$ concentration. The $\mathrm{CO}$ absorption line shape at ambient temperature and a bar pressure given by HITRAN database are used to calculate the Voigt theoretical spectrum. The Voigt theoretical spectrum takes into account the Lorentz lineshape generated by gas collision, spontaneous radiation and Doppler lineshape generated by velocity distribution of luminous particles. The comprehensive function is as follows:

$$
\begin{aligned}
g_{z}\left(v, v_{0}\right) & =\int_{-\infty}^{+\infty} g_{L}\left(v_{1}, v_{0}\right) g_{D}\left(v, v_{1}\right) d v_{1} \\
& =\frac{1}{\pi} \sqrt{\frac{\ln 2}{\pi}} \int_{-\infty}^{+\infty} \frac{\alpha_{L}}{\left(v_{1}-v_{0}\right)^{2}-\alpha_{L}^{2}} \frac{1}{\alpha_{D}} \exp \left(-\omega\left(v_{1}\right)\right) d v_{1}
\end{aligned}
$$

where,

$$
\omega\left(v_{1}\right)=\frac{\ln 2}{\alpha_{D}^{2}}\left(v-v_{1}\right)^{2}
$$

$\alpha_{L}$ and $\alpha_{D}$ are the half-width of Lorentz lineshape and Doppler lineshape, respectively. $v_{0}$ is the central frequency. If $\alpha_{D}$ is set as a constant [19], then:

$$
g_{z}\left(v, v_{0}\right)=\sqrt{\frac{\ln 2}{\pi}} \frac{1}{\alpha_{D}}\left(\frac{\mu}{\pi} \int_{-\infty}^{+\infty} \frac{1}{(\xi-t)^{2}-\mu^{2}} \exp \left(-t^{2}\right) d t\right)
$$

where,

$$
\xi=\sqrt{\ln 2} \frac{v-v_{0}}{\alpha_{D}}, \mu=\sqrt{\ln 2} \frac{\alpha_{L}}{\alpha_{D}}, t=\sqrt{\frac{\ln 2}{\alpha_{D}}}\left(v_{1}-v\right)
$$


The Voigt absorption spectrum has been widely used in differential absorption spectrometry. The MDL of the sensor can be determined by comparing the residual between the spectrum measured in the experiment and the Voigt theoretical spectrum.

\section{Sensor Configuration}

The core device of a gas sensor is a QCL with a center wavelength of $4.65 \mu \mathrm{m}$. The luminous mid-infrared light is focused on the $12 \mathrm{~m}$ long optical path absorption cell (made of stainless steel) through the two reflected-gilt spherical lens. Then, the mid-infrared light emitted from the absorption cell passes through a paraboloid reflector and is received by a liquid nitrogen cooled $\mathrm{HgCdTe}$ detector; the response peak wavelength is $5 \mu \mathrm{m}$, the spectral response range is from $2 \mu \mathrm{m}$ to $12 \mu \mathrm{m}$, the response time is less than $100 \mathrm{~ns}$, and the size of the detection surface is $1 \mathrm{~mm}^{2}$. The overall design block diagram and physical image of the $\mathrm{CO}$ sensor is shown in Figure $2 \mathrm{a}, \mathrm{b}$, respectively.

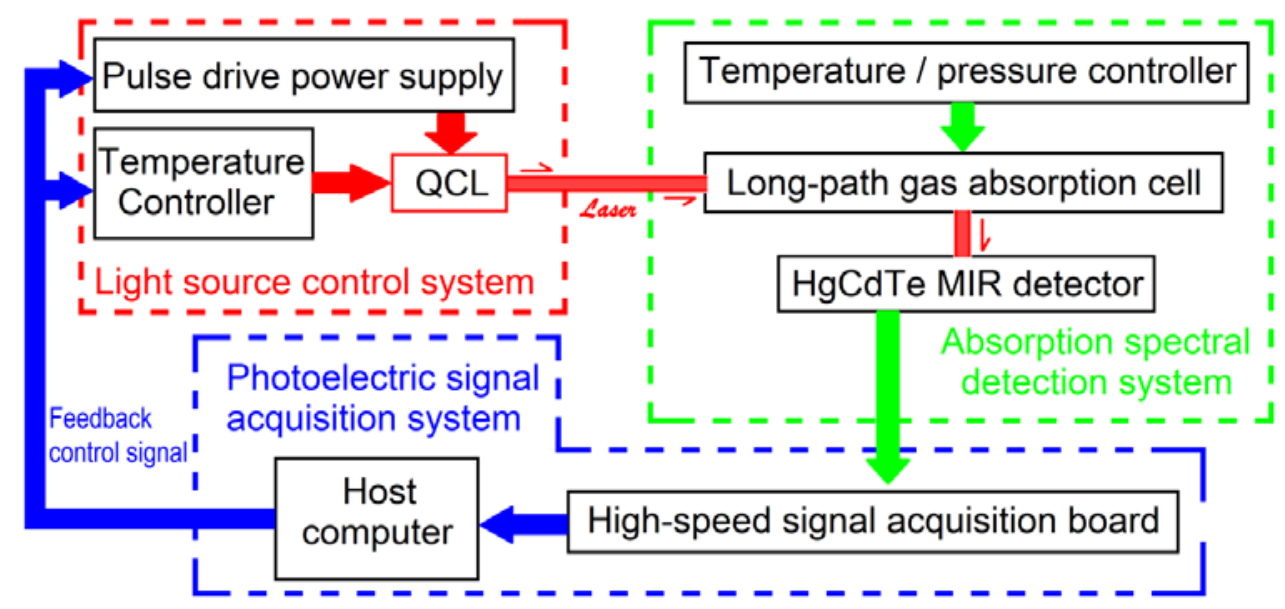

(a)

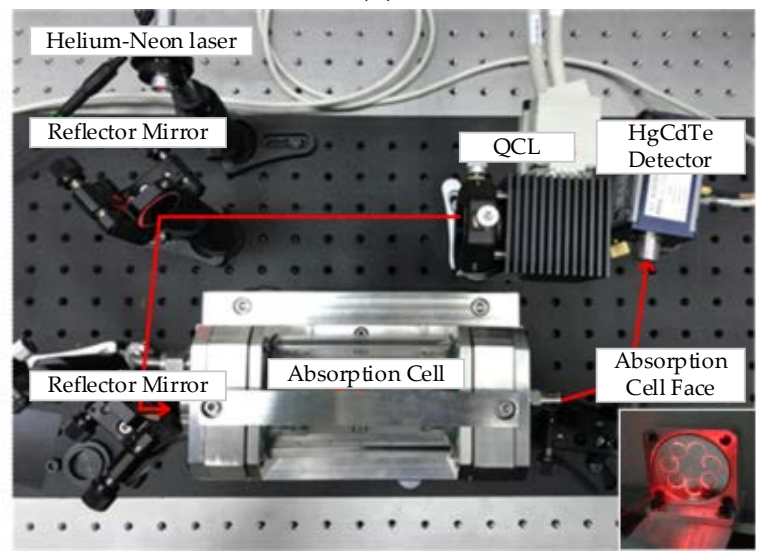

(b)

Figure 2. (a) The block diagram of CO sensor. (b) Physical image of CO sensor.

In the control system, the self-designed constant power controller for QCL is utilized, which is capable of the adjustment of width and duty ratio of the output driving pulse. The peak driving current is $10 \mathrm{~A}$, and the pulse rising/falling time is less than $10 \mathrm{~ns}$. The laser is real-time controlled in a constant power model by reading the feedback current from the photodiode packaged in the inner QCL. The QCL working temperature fluctuation is less than $\pm 0.05^{\circ} \mathrm{C}$ by using adaptive proportional integral derivative (PID) algorithm, and no overshoot control of target working temperature is realized.

The optical part is composed of a gold-plated reflector, multiple reflection mirror, and a mid-infrared $\mathrm{HgCdTe}$ detector. The reflector selects platinum materials as the coating, which have 
a higher than $99 \%$ reflectivity for mid-infrared light. The light path length of the absorption cell used in the sensor is $12 \mathrm{~m}$, the volume is $500 \mathrm{~mL}$, the operating reflection band range is $2.5 \mu \mathrm{m}$ to $10 \mu \mathrm{m}$, and the physical size is $450 \mathrm{~mm} \times 110 \mathrm{~mm} \times 110 \mathrm{~mm}$. Due to the invisibility of mid-infrared, the luminous light emitted from QCL is collimated via a visible red light from the Helium-Neon laser. As a result, most of the mid-infrared light can be reflected through an all-reflecting spherical lens into the inlet aperture of the absorption cell. Finally, optical output signals can be detected by the $\mathrm{HgCdTe}$ detector after several passes in the absorption cell.

\section{Experiment}

Under the conditions of ambient temperature $(293 \mathrm{~K})$ and one bar pressure $\left(1.0 \times 10^{5} \mathrm{~Pa}\right)$, the performance indicators of trace CO sensor, the concentration-voltage response, measurement MDL, and working stability are tested. MDL performance is assessed by comparing the residual difference method and the Allan deviation method.

\subsection{Response}

In order to observe the response of the proposed sensor, $15 \mathrm{CO}$ gas samples with different standard concentrations (10 ppmv to $60 \mathrm{ppmv}$ ) were prepared by dynamic gas dilution equipment. The prepared $\mathrm{CO}$ gases are pumped into the gas cell successively, and the relation between output voltage signal of the sensor and $\mathrm{CO}$ concentration was obtained, as shown in Figure 3.

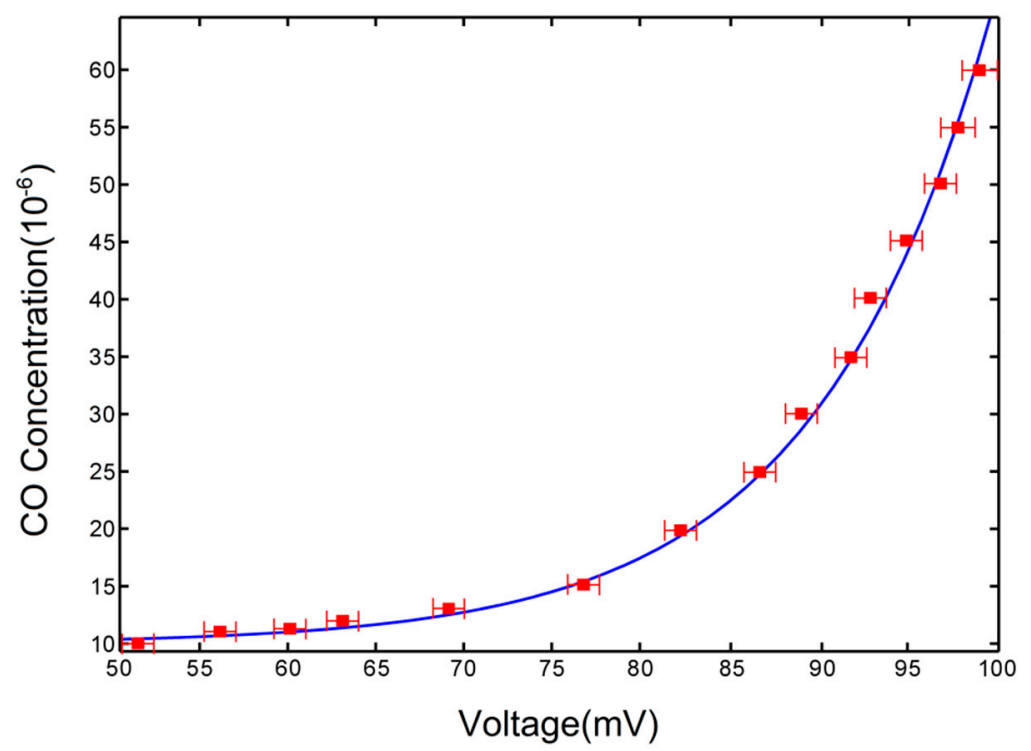

Figure 3. Concentration of $\mathrm{CO}$ versus output voltage of the sensor.

In the figure, the $x$ coordinate represents the output voltage collected by the photoelectric signal acquisition system, the y coordinate describes the concentration of the CO gas sample, and the solid blue line is the fitting curve. It can be seen from the figure that the measured gas concentration with respect to the output voltage is exponential, which is in line with Bill lambert's law, as shown in Formula (1).

\subsection{Stability}

Stability performance can be obtained by measuring the sensor's output changes along with time and a certain concentration of measured gas in absorption cell. The CO gas with a concentration of 60 ppmv was observed for $12 \mathrm{~h}$, and the relationship between the output voltage corresponding to $\mathrm{CO}$ gas concentration and the detection time was recorded; the results are shown in Figure 4. 


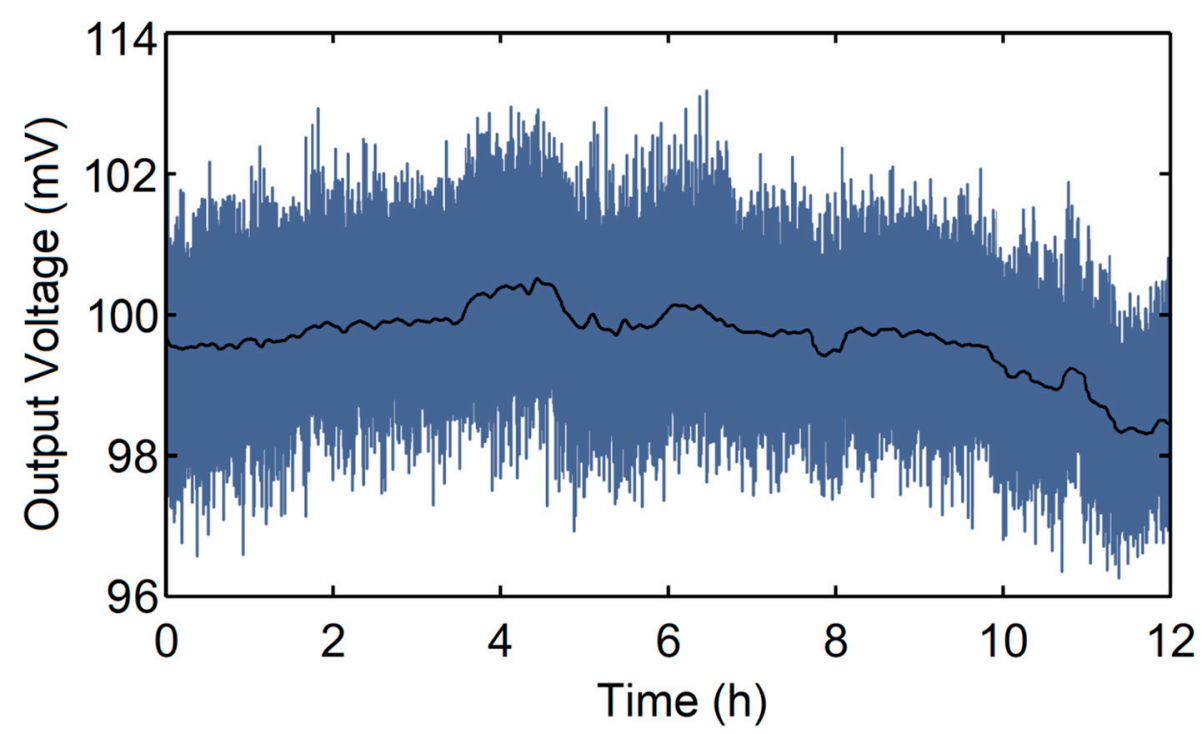

Figure 4. The measured data of 60 ppmv CO over $12 \mathrm{~h}$.

The blue curve is the output voltage collected per second, and the black curve is the average value of the collected data with integral time of $30 \mathrm{~s}$. It can be seen from the figure that the sensor had stable operations within two hours of starting up, the recorded data fluctuated with $0.4 \mathrm{mV}$, and stability was as high as $2.1 \times 10^{-3}$. Accordingly, the response amplitude of the sensor changed within $1.6 \mathrm{mv}$ in $12 \mathrm{~h}$, and the stability of $1.7 \times 10^{-2}$ was achieved in the longer experimental test. In future work, reference cells can adapt to suppress common noise, which leads to stability promotion of the proposed sensor.

\subsection{Minimum Detection Limit}

MDL is defined as the gas concentration when the effective gas absorption signal is distinguishable from background noise, namely when Signal-Noise-Ratio (SNR) is 1. MDL of the proposed sensor was estimated by comparing the residual difference between the measured spectrum and the Voigt theoretical spectrum. The experimental results to measure 10 ppmv CO is shown in Figure 5.

The red curve in Figure 5a is the Voigt theoretical spectrum; the difference curve between the measured absorption spectrum and the base line background is shown below (blue line). The coincidence degree between the Voigt theoretical spectrum and the difference curve is very good, the residual error less than $\pm 0.5 \%$. We investigated the noise spectrum near the absorption peak and found that the noise standard error (SE) is $9.395 \times 10^{-4}$. Here, we can approximately assume that this SE value is the minimum detectable concentration when the Signal-Noise-Ratio (SNR) equals to 1. In addition, the magnitude of absorption spectrum peak is 0.087 , so the $\mathrm{SNR}=0.087 / \mathrm{SE}=92.6$. Due to the measured $\mathrm{CO}$ concentration being $10 \mathrm{ppmv}$, the MDL of the sensor can be calculated as $10 \mathrm{ppmv} / 92.6=108 \mathrm{ppbv}$.

Due to the characteristics of non-stationary and slow-time variation when the sensor worked, we introduced Allan deviation to estimate MDL more accurately. Allan deviation has been widely used in the estimation of MDL of gas sensor [20,21], and its expression is:

$$
\sigma_{A}^{2}(\tau)=\frac{1}{2(N-2) \tau^{2}} \sum_{i=1}^{N-2}\left(X_{i+2}-2 X_{i+1}+X_{i}\right)^{2}
$$

$N$ is the sampling sequence of gas concentration in time domain, $\tau$ is the sampling period, $X_{i}$ is the sampling value. We measured the $\mathrm{CO}$ gas samples of $60 \mathrm{ppmv}$ and calculated the Allan deviation of the acquisition data; the results are shown in Figure 6. 


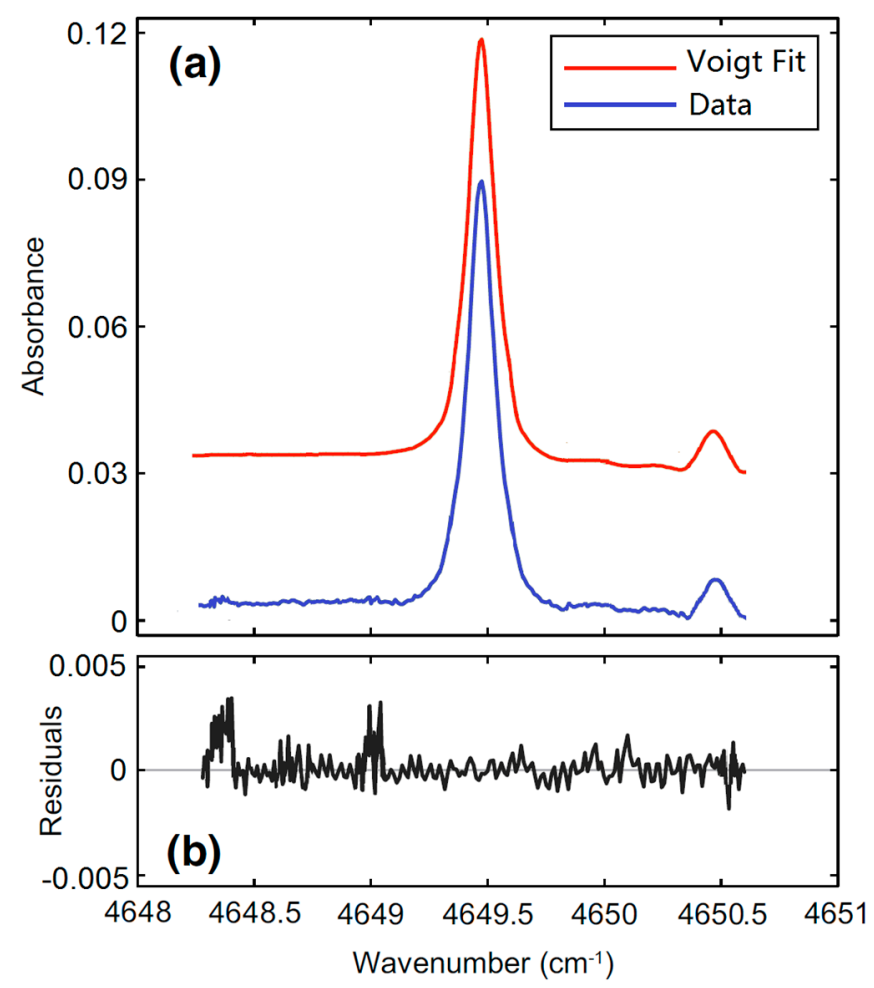

Figure 5. (a) Measured absorption spectrum (blue line) and Voigt theoretical spectrum (red line) via the LOP-DAST method. (b) Residual curve calculated by aforementioned two spectrums.

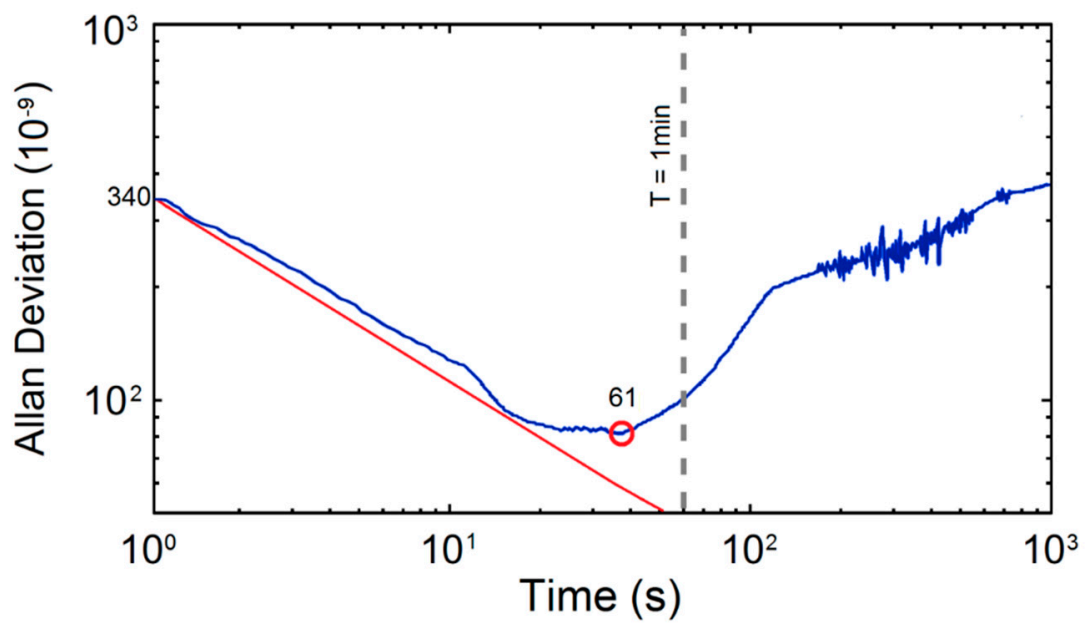

Figure 6. Allan deviation of the acquisition data of $60 \mathrm{ppmv} \mathrm{CO}$.

It can be seen from the figure that the initial value of Allan deviation was about $340 \mathrm{ppbv}$, and the minimum value reached $61 \mathrm{ppbv}$ with integration time of $40 \mathrm{~s}$. This result is also similar to the value obtained from previous residual analyses.

\section{Discussion and Conclusions}

In this paper, a high-performance trace CO sensor using mid-infrared QCL combined with LOP-DAST is introduced. The working principle of LOP-DAST and calculating method of the absorption cross section are described, respectively. The stability of the sensor reached $1.7 \times 10^{-2}$ by testing standard gas sample for a long period, and the MDL achieved approximate 100 ppbv by comparing the residual difference method and the Allan deviation method. Besides, the sensor 
is capable of detecting a variety of trace gases by changing the luminous source with different excitation wavelengths.

Comparing to state-of-the-art trace gas sensors using mid-infrared luminous source, simplified optical structure is utilized to achieve miniaturization and practicality, and maintain high measurement detection performance. Nevertheless, the shortcoming of the bulky package of the optical core devices and the auxiliary modules is obvious, such as bulky electrical power supply and Peltier temperature controller for QCL and $\mathrm{HgCdTe}$ detector, and multi-reflection cell with the size similar to a shoe box. Although the described sensor obtained ppbv levels of MDL, it was too large to be convenient for some special applications with compact volume requirement, such as gas detection in mobile and airborne platforms.

To meet the compact requirements of these specific applications, the challenge of fabrication technique on a chip level is of critical importance. Fortunately, the core components of the described mid-infrared gas sensor have been minimized, such as mid-infrared MEMS luminous source [22], micro-cavity absorption cell using silicon microring resonators [23,24], and on-chip HgCdTe photodiode detectors [25]. The advanced comments combined with integrated packaging technology to constitute new concept sensors on a chip level can potentially rival current infrared absorption spectrum sensors. Thus, micro mid-infrared gas sensors with ultra-compact size and high sensitivity appear to be very promising for future gas sensing.

Author Contributions: C.C. and Y.W. conceived the research and wrote the paper. Q.R., H.P., and P.W. performed the research.

Funding: This research was funded by the National Key R\&D Program of China (2018YFC1503802, 2016YFC0303902), the National Natural Science Foundation of China (61871199), the Science and Technology Department of Jilin Province of China (20180201022GX), and the Education Department of Jilin Province of China (JJKH20180154KJ).

Conflicts of Interest: The authors declare no conflict of interest.

\section{References}

1. Stewart, G.; Mencaglia, A.; Philp, W. Interferometric signals in fiber optic methane sensors with wavelength modulation of the DFB laser source. J. Lightw. Technol. 1998, 16, 43-53. [CrossRef]

2. Wang, W.; Lv, Y. The principal, preparation and application of quantum cascade laser. Laser J. 2018, 39, 7-11.

3. Kourosh, K. Sensors: An Introductory Course; Springer Publications: New York, NY, USA, 2013; p. 29.

4. Chen, J.; Lin, $\mathrm{H}$. The development of $\mathrm{CH}_{4}$ detection technique. Mod. Instrum. 2007, 13, 1-3.

5. $\mathrm{Mu}, \mathrm{Y}$; $\mathrm{Hu}, \mathrm{T}$;; Gong, H.; Ni, R.; Li, $\mathrm{S}$. A Trace $\mathrm{C}_{2} \mathrm{H}_{2}$ Sensor Based on an Absorption Spectrum Technique Using a Mid-Infrared Interband Cascade Laser. Micromachines 2018, 9, 530. [CrossRef] [PubMed]

6. Li, M.; Bai, F. Design of high sensitivity infrared methane detector based on TDLAS-WMS. Laser J. 2018, 39, 75-79.

7. Mcmanus, J.B.; Shorter, J.H.; Nelson, D.D. Pulsed quantum cascade laser instrument with compact design for rapid, high sensitivity measurements of trace gases in air. Appl. Phys. B 2008, 92, 387-392. [CrossRef]

8. Gorrotxategi-Carbajo, P.; Fasci, E.; Ventrillard, I.; Carras, M.; Maisons, G.; Romanini, D. Optical-feedback cavity-enhanced absorption spectroscopy with a quantum-cascade laser yields the lowest formaldehyde detection limit. Appl. Phys. B 2013, 110, 309-314. [CrossRef]

9. Tang, Y.; Liu, W.; Kan, R.; Zhang, Y.; Liu, J.; Xu, Z.; Shu, X.; Zhang, S.; He, Y.; Geng, H.; et al. Spectroscopy processing for the NO measurement based on the room-temperature pulsed quantum cascade laser. Acta Phys. Sin. 2010, 59, 2364-2369.

10. Li, G.; Dong, M.; Song, N.; Song, F.; Zheng, C.; Wang, Y. Carbon monoxide gas detection system based on mid-infrared spectral absorption technique. Spectrosc. Spectr. Anal. 2014, 34, 2839-2844.

11. Wen, Z.; Wang, L.; Chen, G. development and application of quantum cascade laser based gas sensing system. Spectrosc. Spectr. Anal. 2010, 30, 2043-2048.

12. Li, Q.; Wang, Q.; Shang, T. Laser propulsion principle and development. Laser Infrared 2001, 31, 73-75.

13. Zhang, L.; Cui, X. The design of carbon monoxide detector based on tunable diode lasers absorption spectroscope. Laser J. 2014, 35, 54-56. 
14. Sun, Y.; Liu, W.; Wang, S.; Huang, S.; Yu, X. Research on the method of interference correction for nondispersive infrared multi-component gas analysis. Spectrosc. Spectr. Anal. 2011, 31, 2719-2724.

15. Hao, N.; Zhou, B.; Chen, L. Measurement of nitrous acid and retrieval of aerosol parameters with differential optical absorption spectroscopy. Acta Phys. Sin. 2006, 55, 1529-1533.

16. Xu, J.; Xie, P.; Si, F.; Li, A.; Liu, W. Determination of tropospheric $\mathrm{NO}_{2}$ by airborne multi axis differential optical absorption spectroscopy. Acta Phys. Sin. 2012, 61, 024204.

17. Chen, K.; Mei, M. Detection of gas concentrations based on wireless sensor and laser technology. Laser J. 2014, 35, 50-54.

18. Qiao, Y.; Tao, J.; Chen, C.-H.; Qiu, J.; Tian, Y.; Hong, X.; Wu, J. A miniature on-chip methane sensor based on an ultra-low loss waveguide and a micro-ring resonator filter. Micromachines 2017, 8, 160. [CrossRef]

19. Yin, Z.; Wu, C.; Gong, W.; Gong, Z.; Wang, Y. Voigt profile function and its maximum. Acta Phys. Sin. 2013, 62,123301 .

20. Werle, P.; Popov, P. Application of antimonide lasers for gas sensing in the 3-4- $\mathrm{mm}$ range. Appl. Opt. 1999, 38, 1494-1501. [CrossRef] [PubMed]

21. Ye, W.; Zheng, C.; Wang, Y. Stability measurement and temperature compensation of mid-infrared methane detection device. Acta Opt. Sin. 2014, 34, 0323003.

22. Lochbaum, A.; Fedoryshyn, Y.; Dorodnyy, A.; Koch, U.; Hafner, C.; Leuthold, J. On-chip narrowband thermal emitter for mid-IR optical gas sensing. ACS Photonics 2017, 4, 1371-1380. [CrossRef]

23. Khan, H.; Zavabeti, A.; Wang, Y.; Harrison, C.J.; Carey, B.J.; Mohiuddin, M.; Chrimes, A.F.; De Castro, I.A.; Zhang, B.Y.; Sabri, Y.M.; et al. Quasi physisorptive two dimensional tungsten oxide nanosheets with extraordinary sensitivity and selectivity to $\mathrm{NO}_{2}$. Nanoscale 2017, 9, 19162-19175. [CrossRef] [PubMed]

24. Nitkowski, A.; Chen, L.; Lipson, M. Cavity-enhanced on-chip absorption spectroscopy using microring resonators. Opt. Exp. 2008, 16, 11930-11936. [CrossRef]

25. Sun, X.; Abshire, J.B.; Beck, J.D.; Mitra, P.; Reiff, K.; Yang, G. HgCdTe avalanche photodiode detectors for airborne and spaceborne lidar at infrared wavelengths. Opt. Exp. 2017, 25, 16589-16602. [CrossRef] [PubMed] 